\title{
A Cross-sectional Analysis of Patterns and Predictors of Medication Adherence in Bipolar Disorder: Single Center Experience from South India
}

\author{
Nivedhitha Selvakumar, Vikas Menon, Shivanand Kattimani \\ Department of Psychiatry, Jawaharlal Institute of Postgraduate Medical Education and Research (JIPMER), Puducherry, India
}

\begin{abstract}
Objective: Our objective was to determine patterns and predictors of medication adherence in bipolar disorder. Methods: Between August 2015 and December 2016, we recruited 160 patients with a diagnosis of bipolar disorder as per International Classification of Diseases-10: Clinical Descriptions and Diagnostic Guidelines. The diagnosis was further confirmed by using the MINI International Neuropsychiatric Inventory. All of them were currently in remission (confirmed by standard measures) and on stable dosing of medication for at least a year. Medication adherence was assessed using Tamil validated version of Morisky Medication Adherence Scale. Patients were dichotomized into low adherence $(<6)$ and high adherence $(\geq 6)$ groups and compared on various socio-demographic and clinical variables. Results: Majority of the sample $(n=97,60.6 \%)$ demonstrated low adherence to treatment regimen. Being employed and having spent greater number of days in hospital were predictive of higher medication adherence (odds ratio [OR] 2.78, 95\% confidence interval $[\mathrm{Cl}]$ 1.019-7.585; and OR 1.02, 95\% Cl 1.003-1.037, respectively). Fewer number of lifetime depressive episodes and positive drug attitudes demonstrated trend level positive association with high medication adherence.

Conclusion: Non-adherence to prescribed medications is a common problem in bipolar disorder. Interventions targeting vocation, medication focused psychoeducation and promotion of positive drug attitudes are likely to enhance medication adherence in this group.
\end{abstract}

KEY WORDS: Bipolar disorder; Medication adherence; Medication compliance; Medication persistence; Therapeutics; Psychiatry.

\section{INTRODUCTION}

The World Health Organization has defined treatment adherence as: "The extent to which a person's behaviortaking medication, following a diet, and/or executing lifestyle changes, corresponds with agreed recommendations from a healthcare provider". ${ }^{1)}$ Bipolar disorder (BD) is a highly prevalent, chronic and disabling condition. Among the many unmet needs in managing this condition, improving adherence to medications is a clear and

Received: January 25, 2017 / Revised: March 10, 2017

Accepted: April 3, 2017

Address for correspondence: Vikas Menon, MD

Department of Psychiatry, Jawaharlal Institute of Postgraduate Medical Education and Research (JIPMER), Puducherry-605006, India

Tel: +91-413-2276265, Fax: +91-413-2272067

E-mail: drvmenon@gmail.com

ORCID: https://orcid.org/0000-0001-8035-4658 present challenge to health care providers. ${ }^{2,3)}$ In a widely quoted study on 13-year outcomes in $\mathrm{BD}$, the authors found that bipolar subjects spent about $32 \%$ of time in clinically significant depression, $9 \%$ with manic symptoms and $6 \%$ with symptoms from both poles. ${ }^{4)}$ Medication non-adherence is often a greater problem in psychiatric disorders as compared to other chronic medical conditions and has been postulated as a possible reason for the high residual symptom burden in $\mathrm{BD} .{ }^{5,6)}$

Existing literature suggests that about $20-60 \%$ of BD patients are non-adherent to their medication regimens. ${ }^{7,8)}$ Further, non-adherence has been shown to have a robust association with a variety of negative outcomes such as relapse, re-hospitalizations, sub-optimal functioning and suicidality in BD. ${ }^{9-11)}$ Many authors also argue that nonadherence is the reason for the "efficacy-effectiveness" gap seen in $\mathrm{BD}$, wherein an increased response to drug

(c) This is an Open-Access article distributed under the terms of the Creative Commons Attribution Non-Commercial License (http://creativecommons.org/licenses/by-nc/4.0) which permits unrestricted non-commercial use, distribution, and reproduction in any medium, provided the original work is properly cited. 
treatments is seen in clinical trials than in real world practice. ${ }^{12,13)}$ Consequently, it is important to systematically investigate the determinants of medication adherence so that clinicians and health care providers can work on these factors to optimize management. A range of factors have been found to be predictive of non-adherence in bipolar subjects including concurrent alcohol use disorder, greater severity of depressive episodes, polypharmacy, negative attitudes towards medication, quality of doctor-patient relationship and decreased insight. ${ }^{7,8,14-17)}$

Evidence suggests that adherence is a complex behavior that is a result of an interaction of several variables such as patient attributes about illness, social context, access to treatment and service delivery ${ }^{1,18-20)}$ which may presumably differ across cultures and settings. In this scenario, we carried out the present study with two objectives: first, to assess medication adherence patterns using a self-report measure validated in the local language and second, to identify predictors of medication adherence in BD among South Indian Tamil speaking population.

\section{METHODS}

The present cross-sectional study was carried out in the Department of Psychiatry in a teaching cum tertiary care hospital over a period of one and a half years from August 2015 to December 2016. The hospital is located in Puducherry, South India and Tamil is the local language. The study had prior approval from JIPMER Institute Human Ethics Committee via approval No. JIP/IEC/2015/16/603. Subjects were recruited from the weekly outpatient Mood Disorders Clinic run by the Department of Psychiatry. All patients with a mood spectrum disorder diagnosis, treated either on in-patient or out-patient basis, are eventually assigned to this clinic for getting their regular drug refills. Patients are asked to follow up once every three weeks for their regular check-up and refills of their medicines. Checkups and medicine refills are provided free of cost through governmental aid. Psychotropic agents stocked in the hospital pharmacy include three antipsychotics (haloperidol, chlorpromazine, and risperidone), three mood stabilizers (lithium, sodium valproate, and carbamazepine), two anti-depressants (fluoxetine and amitryptiline) and two anxiolytics (diazepam and clonazepam). These drugs are made available throughout the year for free dispensing in order to benefit the patients.
Consequently, nearly every patient in the follow-up clinic is managed with agents available from this basket of drugs. Presently, the clinic has about 400 to 500 patients on its rolls and diagnoses are made as per International Classification of Diseases-10: Clinical descriptions and diagnostic guidelines. ${ }^{21)}$

The inclusion criteria for the present study were: patients in the age group 18-65 years with a file diagnosis of Bipolar Affective Disorder as per International Classification of Diseases-10: Clinical Descriptions and Diagnostic Guidelines (F31.0-F31.7). ${ }^{21)}$ Additionally, the MINI International Neuropsychiatric Interview (MINI) was used to confirm the diagnosis. ${ }^{22)}$ We included only patients on stable dosing of medications for the past one year and in clinical remission (Hamilton Depression Rating Scale $[\mathrm{HDRS}]^{23)}$ scores $\leq 7 /$ Young Mania Rating Scale $\left[\right.$ YMRS] ${ }^{24)}$ scores $\leq 8$ ). All such patients, who were registered into the follow-up clinic for continued care, were screened for eligibility regardless of their compliance with prescribed drugs. Those on stable dosing of medications were selected as it was deemed unethical to include other patients. Participants were selected by convenient sampling from the outpatient Department of Psychiatry after applying the inclusion/exclusion criteria and obtaining written informed consent for participation.

Basic and relevant socio-demographic data was collected using a semi-structured proforma. Details about physical and psychiatric co-morbidity were collected from patient and informant interviews as well as verified from available records. Subsequently, all patients were rated on the following measures;

1. Morisky Medication Adherence Scale (MMAS)- ${ }^{25}$ : This is a structured, widely used, self-reported measure for medication adherence, the primary outcome measure for this study. It has better psychometric properties than the 4-item Morisky, Green and Levine adherence scale, particularly a higher internal consistency (Cronbach's alpha of 0.83 vs. 0.61$){ }^{25,26)}$ The scale has been used for a range of chronic health conditions, including BD. ${ }^{27,28)}$ For the study, we obtained permission to use the Tamil translated and validated version of MMAS-8. The scale consists of eight questions. The first seven elicit a dichotomous reply (yes/no) and the last one is answered using a 5-point Likert Scale. Total score ranges from 0 to 8 with three categorieslow adherence $(<6)$, medium adherence $(\geq 6$ but $<8$ ) and high adherence (score of 8 ). Since only very few pa- 
tients ( $n=12)$ reported high adherence, we dichotomized the dependent variable into two groups-low adherence (score $<6$ ) and high adherence (score $\geq 6$ ).

2. Drug Attitudes Inventory (DAl)- $10^{29)}$ : This is a 10-item shorter version of the original DAI-30 designed to assess attitudes, experiences and beliefs about psychiatric medications. Each item is designed to elicit a dichotomous response (true or false). Some items are reverse scored. Total score is obtained by summing the individual items and ranges from -10 to +10 . Patients were categorized as having negative attitudes (total score $<10$ ) or positive attitudes (total score $>10$ ) in line with the original scoring method. ${ }^{30}$

\section{Statistical Analysis}

Data were analyzed using IBM SPSS Statistics version 20 (IBM Co., Armonk, NY, USA). Baseline socio-demographic and clinical variables were represented as mean with standard deviation and frequencies and percentages for continuous and categorical data respectively. They were compared between the low and high medication adherence groups (defined earlier) in the univariate analysis. Strength of these comparisons was measured with nonparametric Mann-Whitney $U$ test or Student $t$ test for nonnormal and normally distributed variables respectively and chi-square test for discrete variables. Normality was assessed using the Shapiro-Wilk test. For identifying predictors of medication adherence, variables which showed at least a trend level significance in the univariate analysis $(p<0.10)$ were entered into the backward logistic regression model. This method, where all variables are entered into the model in the first step and then sequentially deleted, was used as the study was exploratory in nature and gives more chance for variables to remain in the final regression equation. All statistical analyses were carried out for two-tailed significance and a $p<0.05$ was considered significant for all univariate and multivariate comparisons.

\section{RESULTS}

The sample comprised of 160 patients with BD. The mean age of the sample was $37.8 \pm 10.8$ years (range, $18-64$ years). The mean age of males was $37.7 \pm 11.3$ years and females were $37.9 \pm 10.4$. Males $(50.6 \%)$ and females $(49.4 \%)$ were nearly equally represented in the sample.
Majority of the sample $(72.5 \%)$ were married and had studied till tenth grade $(68.1 \%)$. The total number of lifetime episodes varied from 1 to 20 . Overall, there were more lifetime manic episodes (mean, 3.4; standard deviation $[\mathrm{SD}], 2.6)$ than depressive (mean, $0.7 ; \mathrm{SD}, 1.4$ ). Nearly three-fourth of the sample $(n=118,73.8 \%)$ had received in-patient care for their mood episodes at least once during the course of their illness. The other baseline characteristics are shown in Table 1.

Table 1. Baseline socio-demographic characteristics of the entire sample

\begin{tabular}{|c|c|}
\hline Variable & Data \\
\hline \multicolumn{2}{|l|}{ Age (yr) } \\
\hline $18-25$ & $23(14.4)$ \\
\hline $26-35$ & $46(28.8)$ \\
\hline $36-45$ & $52(32.5)$ \\
\hline $46-55$ & $29(18.1)$ \\
\hline$\geq 56$ & $10(6.3)$ \\
\hline \multicolumn{2}{|l|}{ Sex } \\
\hline Male & $81(50.6)$ \\
\hline Female & $79(49.4)$ \\
\hline \multicolumn{2}{|l|}{ Marital status } \\
\hline Married & $116(72.5)$ \\
\hline Single & $44(27.5)$ \\
\hline \multicolumn{2}{|l|}{ Education } \\
\hline$\leq 10$ th grade & $109(68.1)$ \\
\hline$>10$ th grade & $51(31.9)$ \\
\hline \multicolumn{2}{|l|}{ Occupation } \\
\hline Employed & $87(54.4)$ \\
\hline Not employed & $73(45.6)$ \\
\hline \multicolumn{2}{|l|}{ Current substance dependence* } \\
\hline Yes & $18(11.3)$ \\
\hline No & $140(87.5)$ \\
\hline \multicolumn{2}{|c|}{ Family history of psychiatric illness } \\
\hline Yes & $69(43.1)$ \\
\hline No & $91(56.9)$ \\
\hline \multicolumn{2}{|l|}{ History of chronic medical illness } \\
\hline Yes & 35 (21.9) \\
\hline No & $125(78.1)$ \\
\hline Duration of illness (yr) & $13.0 \pm 8.6$ \\
\hline Age at onset (yr) & $24.8 \pm 8.4$ \\
\hline Lifetime episodes & $4.1 \pm 3.1$ \\
\hline Manic episodes & $3.4 \pm 2.6$ \\
\hline Depressive episodes & $0.7 \pm 1.4$ \\
\hline In-patient admissions & $1.7 \pm 1.9$ \\
\hline Total of hospital days & $48.7 \pm 66.8$ \\
\hline YMRS score & $0.43 \pm 0.7$ \\
\hline HDRS score & $0.96 \pm 0.9$ \\
\hline Drug Attitudes Inventory score & $7.5 \pm 2.1$ \\
\hline
\end{tabular}

Values are presented as number (\%) or mean \pm standard deviation. YMRS, Young Mania Rating Scale; HDRS, Hamilton Depression Rating Scale.

*Data missing for two cases. 
With regard to concurrent physical illness, diabetes mellitus was the single most common medical co-morbidity ( $n=22)$ followed by systemic hypertension $(n=6)$, hypothyroidism $(n=5)$, bronchial asthma $(n=4)$, tuberculosis $(n=2)$, obesity $(n=1)$ and psoriasis $(n=1)$. Overall, 35 patients $(21.9 \%$ ) had a history of chronic medical illness of whom six patients had multiple medical comorbidities. All patients diagnosed with diabetes in our sample were on oral hypoglycemic agents (either tablet [Tab.] metformin alone [1,000-1,500 mg per day] or combination of Tab. metformin plus Tab. glibenclamide). Two of the patients diagnosed with systemic hypertension were on calcium channel blockers (Tab. amlodipine 5 mg) while others were not taking any treatment for the same. Similarly, none of the bronchial asthma patients were on any long term medications while all patients with hypothyroidism were on replacement thyroxin therapy (50-100 $\mu$ g per day). Both the patients with tuberculosis had recovered and were not on any active treatment for the same. No treatment details were available for five patients. Regarding psychiatric co-morbidity, 18 patients $(11.3 \%)$ fulfilled criteria for substance dependence while another 10 patients $(6.3 \%)$ were using substances in a harmful manner. Two patients (1.3\%) had evidence of

Table 2. Correlates of medication adherence

\begin{tabular}{|c|c|c|c|}
\hline Variable & High adherence group (n=63) & Low adherence group $(n=97)$ & Comparison ( $p$ value) \\
\hline Age (yr) & $38.3 \pm 10.9$ & $36.9 \pm 10.7$ & $t=-0.779(0.437)$ \\
\hline Gender & & & $\chi^{2}=0.465(0.495)$ \\
\hline Male & $34(56)$ & $47(48.5)$ & \\
\hline Female & $29(44)$ & $50(51.5)$ & \\
\hline Marital status & & & $\chi^{2}=0.014(0.906)$ \\
\hline Married & $46(73.0)$ & $70(72.2)$ & \\
\hline Single & $17(27.0)$ & $27(27.8)$ & \\
\hline Education & & & $\chi^{2}=0.444(0.505)$ \\
\hline$\leq 10$ th grade & $41(65.1)$ & $68(70.1)$ & \\
\hline$>10$ th grade & $22(34.9)$ & $29(29.9)$ & \\
\hline Occupation & & & $\chi^{2}=3.482(0.062)$ \\
\hline Employed & $40(63.5)$ & $47(48.5)$ & \\
\hline Not employed & $23(36.5)$ & $50(51.5)$ & \\
\hline Substance dependence* & & & $\chi^{2}=1.120(0.290)$ \\
\hline Yes & $5(7.9)$ & $13(13.4)$ & \\
\hline No & $57(90.5)$ & $83(85.6)$ & \\
\hline Family history of psychiatric illness & & & $\chi^{2}=0.358(0.550)$ \\
\hline Yes & $29(46.0)$ & $40(41.2)$ & \\
\hline No & $34(54.0)$ & $57(58.8)$ & \\
\hline History of chronic medical illness & & & $\chi^{2}=1.185(0.276)$ \\
\hline Yes & $11(17.5)$ & $24(24.7)$ & \\
\hline No & $52(82.5)$ & $73(75.3)$ & \\
\hline Polypharmacy & & & $\chi^{2}=0.215(0.643)$ \\
\hline Yes & $36(57.1)$ & $59(60.8)$ & \\
\hline No & $27(42.9)$ & $38(39.2)$ & \\
\hline Duration of illness (yr) & $12.9 \pm 9.6$ & $13.1 \pm 7.9$ & $U=2,901.00(0.589)$ \\
\hline Age at onset (yr) & $24.0 \pm 8.0$ & $25.3 \pm 8.6$ & $U=2,823.50(0.417)$ \\
\hline Lifetime episodes & $3.8 \pm 2.9$ & $4.2 \pm 3.2$ & $U=2,768.00(0.306)$ \\
\hline Manic episodes & $3.2 \pm 2.6$ & $3.5 \pm 2.6$ & $U=2,757.00(0.287)$ \\
\hline Depressive episodes & $0.4 \pm 0.8$ & $0.8 \pm 1.7$ & $\mathrm{U}=2,742.50(0.240)$ \\
\hline In-patient admissions & $1.7 \pm 1.8$ & $1.8 \pm 1.9$ & $U=3,014.50(0.883)$ \\
\hline Total of hospital days & $51.4 \pm 58.2$ & $46.9 \pm 72.0$ & $U=2,785.00(0.340)$ \\
\hline YMRS score & $0.51 \pm 0.7$ & $0.38 \pm 0.7$ & $U=2,725.50(0.168)$ \\
\hline HDRS score & $0.86 \pm 0.9$ & $1.02 \pm 0.9$ & $U=2,776.50(0.302)$ \\
\hline Drug Attitudes Inventory score & $8.0 \pm 1.7$ & $7.2 \pm 2.3$ & $\mathrm{t}=2.165(0.032)^{*}$ \\
\hline
\end{tabular}

Values are presented as mean \pm standard deviation or number (\%).

YMRS, Young Mania Rating Scale; HDRS, Hamilton Depression Rating Scale.

*Data missing for two cases.

Comparisons made using Student $t$ test $(\mathrm{t})$ or chi-square $\left(\chi^{2}\right)$ or Mann-Whitney $U$ test $(U) ;{ }^{*} p<0.05$. 
paranoid personality traits. No psychiatric co-morbidity was found in the remaining sample ( $n=130,81.3 \%)$.

Ninety-seven patients (60.6\%) demonstrated low adherence to the treatment regimen. Medium adherence was noted in 51 patients (31.9\%) while only 12 patients (7.5\%) had high adherence. Next, we conducted a univariate analysis to identify variables that differed between the dichotomized medication adherence groups (Table 2). It was observed that none of the socio-demographic or clinical variables differed between the groups apart from drug attitude scores which were higher (reflecting more positive attitudes) in the high medication adherence group. Further, the residual mood state (measured using HDRS and YMRS scales) did not distinguish between the high and low medication adherence groups (Table 2).

Subsequently, we did a multivariate analysis using binary logistic regression to find out the predictors of high medication adherence (dependent variable). Co-variates studied included age, gender, occupational status and illness characteristics such as total number of lifetime episodes, number of manic episodes, number of depressive episodes and total number of days spent in hospitalization. Most of these co-variates had a $p<0.3$ in the univariate analysis and hence were included in the regression model. Drug attitudes were also included as a co-variate as it had emerged significant in the univariate analysis. Table 3 summarizes the results of the regression analysis. It was observed that occupational status and length of hospital stay were predictive of high medication adherence. Drug attitude scores and number of depressive episodes had a positive and negative trend level association with high medication adherence respectively. These variables put together explained $22.6 \%$ of the variance in medication adherence (Nagelkerke $\mathrm{R}$ square of model=0.226). Hosmer-Lemeshow goodness of fit statistic was 5.498 ( $p=0.703)$ indicating that the regression

Table 3. Summary of logistic regression model for predictors of medication adherence

\begin{tabular}{lccl}
\hline \multicolumn{1}{c}{ Variable } & $\operatorname{Exp}(\mathrm{B})$ & $95 \% \mathrm{Cl}$ for B & $p$ value \\
\hline Occupation & 2.780 & $1.019-7.585$ & $0.046^{*}$ \\
Number of depressive episodes & 0.216 & $0.039-1.190$ & 0.078 \\
Number of days spent in hospital & 1.020 & $1.003-1.037$ & $0.021^{*}$ \\
Drug Attitude Inventory scores & 1.227 & $1.000-1.506$ & 0.050 \\
\hline
\end{tabular}

$\mathrm{Cl}$, confidence interval.

*Significant at $p<0.05$. model was acceptable.

\section{DISCUSSION}

The present study found that majority of patients $(60.6 \%)$ with BD had low adherence to their medication regimen and this is roughly consistent with previous studies in this disorder. ${ }^{28,31,32)}$ Only very few patients $(7.5 \%)$ in our study were fully adherent. Taking into account the fact that this was a naturalistic descriptive study, the results are a grim reminder that non-adherence continues to be a major issue among bipolar subjects. Clinicians need to consider this when planning management as non-adherence to medications can compromise clinical outcomes. Majority of our sample ( $n=93,58.1 \%)$ were on two psychotropic agents (most commonly mood stabilizer plus antipsychotic) and very few were on more than two agents $(n=3,1.9 \%)$. Hence, we could not examine potential relationships between increasing treatment regimen complexity and medication adherence. This is an area for further exploration.

Regarding predictors of medication adherence, we noted that those who had high adherence were likely to be employed and had spent higher number of days in the hospital across their admissions. Such individuals were also noted to have more positive attitudes towards their medications and had fewer lifetime depressive episodes though these two factors had a trend level significance. There is some variation in factors predictive of medication adherence in published literature on BDs. One study found an overrepresentation of young, unmarried and socially isolated individuals among bipolar patients who are non-adherent to medications ${ }^{33)}$ while another reported male gender, higher educational status, no substance use and monotherapy as factors associated with treatment adherence in this group. ${ }^{28)}$ Studies on non-association of adherence with gender have also been published. ${ }^{14)}$ Echoing our findings, lesser work impairment was noted as a correlate of medication adherence by Kutzelnigg et al. ${ }^{34)}$ but the same study also observed a negative association of adherence with duration of in-patient care. However, the authors have mentioned that their sample could have been biased as they only included patients stabilized on olanzapine. Higher duration of in-patient satay offers more opportunities for psychoeducation and clarification of medication related risk-benefit tradeoff. This may ex- 
plain our findings.

Positive attitudes towards medications have been robustly linked to medication adherence earlier and our study adds to these findings. ${ }^{35,36)}$ It may be argued that better attitudes could be an epiphenomenon of increased hospital stay but our multivariate analysis showed that positive attitudes towards medication can predict medication adherence even after controlling for hospital stay. Substance use has been shown to influence medication adherence in $\mathrm{BD}$ and also play a key role in the relationship between drug attitudes and medication adherence. $^{37-39)}$ However, only a very small percentage of our sample had current substance use disorder (SUD; $11.2 \%$ ) and this may be the reason for non-significant role of substance use in our sample. Nevertheless, many BD patients, irrespective of SUD, deny any illness and disagree with doctors about need for medications. ${ }^{40,41)}$ This means that non-adherence is a serious challenge even in the absence of SUD. We also found that patients with negative drug attitudes at baseline may represent a subgroup at high risk for medication non-adherence and poor outcomes. This has obvious implications from a management perspective. Proper psychoeducation about the benefits of psychotropic medications may address denial of illness. Further, involvement of community health care facilities may surmount lack of access to continued treatment facilities which has been noted to be a reason for non-adherence among Indian patients with schizophrenia ${ }^{42)}$ and may also be relevant to BD.

The major limitations of the study include its cross-sectional design and lack of objective measures to measure medication adherence such as pill count or blood level estimation. Moreover, the entire sample was drawn from a single tertiary care hospital and the results may not necessarily generalize to other settings. Pill counts could not be done as most patients do not come to the follow up clinic with pill boxes or medicine strips unless informed beforehand and this being a cross-sectional study, there was no scope for repeated evaluations. Another limitation is that we have not assessed difference in adherence rates between various mood stabilizers and antipsychotics (first generation vs second generation) separately. We did not contact patients who may have dropped out of the clinic prior to enrollment period of study as we did not have their contact details. The major strength of the study was the use of a validated self-report measure in the local lan- guage to assess medication adherence patterns. To reduce degree of diagnostic imprecision, we also used the MINI instrument to confirm the lifetime diagnosis of BD. Further, the study had a naturalistic design and therefore, the findings are likely to reflect real world practice.

To conclude, majority of stable and remitted bipolar subjects are poorly adherent with their drug treatment regimens. Those who are employed and those who have spent a higher duration as an in-patient are more likely to be medication adherent as are those who have better drug attitudes and experienced fewer lifetime depressive episodes. Other socio-demographic and illness variables appear to be less helpful in determining medication adherence. Clinicians should focus on providing targeted psychoeducation about illness and more specifically, medication related benefits to BD subgroups such as those with poor attitudes to improve adherence. Other BD subgroups at potential risk of non-adherence such as those with substance abuse and personality disorders need further evaluation. The role of technology in improving medication adherence also warrants evaluation in $\mathrm{BD}$ given that simple, low cost mobile health initiatives such as text messages have been found to be beneficial in improving medication adherence in a wide range of conditions including mental health disorders. ${ }^{43-45)}$

\section{- Acknowledgments}

The manuscript is drawn from a thesis for the award of postgraduate degree in Psychiatry. The authors gratefully acknowledge funding support received from JIPMER Intramural research fund (vide Circular No JIP/Res/IntraMD-MS/01/2015-16) that went towards procuring the Tamil validated version of the Morisky Medication Adherence Scale from the original developers. Special thanks are also due to the scale developers and copyright holders for permitting us to use the tool for this research. The authors report no conflict of interest.

\section{REFERENCES}

1. World Health Organization. Adherence to long term therapy: evidence for action. Geneva:WHO;2003.

2. Byrne N, Regan C, Livingston G. Adherence to treatment in mood disorders. Curr Opin Psychiatry 2006; 19:44-49.

3. Ketter TA. Nosology, diagnostic challenges, and unmet needs in managing bipolar disorder. J Clin Psychiatry 2010;71:e27.

4. Judd LL, Akiskal HS, Schettler PJ, Endicott J, Maser J, Solomon DA, et al. The long-term natural history of the weekly sympto- 
matic status of bipolar I disorder. Arch Gen Psychiatry 2002; 59:530-537.

5. Cramer JA, Rosenheck R. Compliance with medication regimens for mental and physical disorders. Psychiatr Serv 1998;49:196-201.

6. Belzeaux R, Correard N, Boyer L, Etain B, Loftus J, Bellivier F, et al. Depressive residual symptoms are associated with lower adherence to medication in bipolar patients without substance use disorder: results from the FACE-BD cohort. J Affect Disord 2013;151:1009-1015.

7. Lingam R, Scott J. Treatment non-adherence in affective disorders. Acta Psychiatr Scand 2002;105:164-172.

8. Colom F, Vieta E, Tacchi MJ, Sánchez-Moreno J, Scott J. Identifying and improving non-adherence in bipolar disorders. Bipolar Disord 2005;7 Supp/ 5:24-31.

9. Strakowski SM, Keck PE Jr, McElroy SL, West SA, Sax KW, Hawkins JM, et al. Twelve-month outcome after a first hospitalization for affective psychosis. Arch Gen Psychiatry 1998; 55:49-55.

10. Altman S, Haeri S, Cohen LJ, Ten A, Barron E, Galynker II, et al. Predictors of relapse in bipolar disorder: a review. I Psychiatr Pract 2006;12:269-282.

11. Gonzalez-Pinto A, Mosquera F, Alonso M, López P, Ramírez $\mathrm{F}$, Vieta E, et al. Suicidal risk in bipolar / disorder patients and adherence to long-term lithium treatment. Bipolar Disord 2006;8:618-624.

12. Schou M. The combat of non-compliance during prophylactic lithium treatment. Acta Psychiatr Scand 1997;95:361-363.

13. Scott J. Using Health Belief Models to understand the efficacy-effectiveness gap for mood stabilizer treatments. Neuropsychobiology 2002;46 Suppl 1:13-15.

14. Baldessarini RJ, Perry R, Pike J. Factors associated with treatment nonadherence among US bipolar disorder patients. Hum Psychopharmacol 2008;23:95-105.

15. Sajatovic M, Bauer MS, Kilbourne AM, Vertrees JE, Williford W. Self-reported medication treatment adherence among veterans with bipolar disorder. Psychiatr Serv 2006;57:56-62.

16. Scott J, Pope M. Nonadherence with mood stabilizers: prevalence and predictors. J Clin Psychiatry 2002;63:384-390.

17. Johnson FR, Ozdemir S, Manjunath R, Hauber AB, Burch SP, Thompson TR. Factors that affect adherence to bipolar disorder treatments: a stated-preference approach. Med Care 2007:45:545-552.

18. Jimmy B, Jose J. Patient medication adherence: measures in daily practice. Oman Med J 2011,26:155-159.

19. Haynes RB, Devereaux PJ, Guyatt GH. Physicians' and patients' choices in evidence based practice. BMJ 2002;324: 1350.

20. Haynes RB, McKibbon KA, Kanani R. Systematic review of randomised trials of interventions to assist patients to follow prescriptions for medications. Lancet 1996;348:383-386.

21. World Health Organization. The ICD-10 classification of mental and behavioural disorders: clinical description and di- agnostic guidelines. Geneva:WHO;1992.

22. Sheehan DV, Lecrubier $\mathrm{Y}$, Sheehan $\mathrm{KH}$, Amorim $\mathrm{P}$, Janavs J, Weiller E, et al. The Mini-International Neuropsychiatric Interview (M.I.N.I.): the development and validation of a structured diagnostic psychiatric interview for DSM-IV and ICD-10. J Clin Psychiatry 1998;59 Suppl 20:22-33;quiz 34-57.

23. Hamilton M. A rating scale for depression. J Neurol Neurosurg Psychiatry 1960;23:56-62.

24. Young RC, Biggs JT, Ziegler VE, Meyer DA. A rating scale for mania: reliability, validity and sensitivity. $\mathrm{Br} / \mathrm{P}$ sychiatry 1978;133:429-435.

25. Morisky DE, Ang A, Krousel-Wood M, Ward HJ. Predictive validity of a medication adherence measure in an outpatient setting. I Clin Hypertens (Greenwich) 2008;10:348-354.

26. Morisky DE, Green LW, Levine DM. Concurrent and predictive validity of a self-reported measure of medication adherence. Med Care 1986;24:67-74.

27. Lam WY, Fresco P. Medication adherence measures: an overview. Biomed Res Int 2015;2015:217047.

28. Bates JA, Whitehead R, Bolge SC, Kim E. Correlates of medication adherence among patients with bipolar disorder: results of the bipolar evaluation of satisfaction and tolerability (BEST) study: a nationwide cross-sectional survey. Prim Care Companion J Clin Psychiatry 2010;12:PCC.09m00883.

29. Hogan T, Awad A. Drug attitude inventory. In: Rush Al, editor. Handbook of psychiatric measures. Arlington, VA:American Psychiatric Association Publishing;2000.

30. Hogan TP, Awad AG, Eastwood R. A self-report scale predictive of drug compliance in schizophrenics: reliability and discriminative validity. Psychol Med 1983;13:177-183.

31. Sajatovic M, Valenstein M, Blow F, Ganoczy D, Ignacio R. Treatment adherence with lithium and anticonvulsant medications among patients with bipolar disorder. Psychiatr Serv 2007;58:855-863.

32. Sajatovic M, Valenstein M, Blow FC, Ganoczy D, Ignacio RV. Treatment adherence with antipsychotic medications in bipolar disorder. Bipolar Disord 2006;8:232-241.

33. Perlick DA, Rosenheck RA, Kaczynski R, Kozma L. Medication non-adherence in bipolar disorder: A patient-centered review of research findings. Clin Approaches Bipolar Disord 2004;3:56-64.

34. Kutzelnigg A, Kopeinig M, Chen CK, Fábián A, Pujol-Luna $\mathrm{MG}$, Shin YC, et al. Compliance as a stable function in the treatment course of bipolar disorder in patients stabilized on olanzapine: results from a 24-month observational study. Int J Bipolar Disord 2014;2:13.

35. Teter CJ, Falone AE, Bakaian AM, Tu C, Ongür D, Weiss RD. Medication adherence and attitudes in patients with bipolar disorder and current versus past substance use disorder. Psychiatry Res 2011;190:253-258.

36. Lee LT, Chen KC, Chang WH, Chen PS, Lee IH, Yang YK. Holistic consideration of patients with schizophrenia to improve medication adherence and outcomes. Clin Psycho- 
pharmacol Neurosci 2015;13:138-143.

37. Perlis RH, Ostacher MJ, Miklowitz DJ, Hay A, Nierenberg AA, Thase ME, et al. Clinical features associated with poor pharmacologic adherence in bipolar disorder: results from the STEP-BD study. J Clin Psychiatry 2010;71:296-303.

38. Keck PE Jr, McElroy SL, Strakowski SM, Bourne ML, West SA. Compliance with maintenance treatment in bipolar disorder. Psychopharmacol Bull 1997;33:87-91.

39. Weiss RD, Ostacher MJ, Otto MW, Calabrese JR, Fossey M, Wisniewski SR, et al; for STEP-BD Investigators. Does recovery from substance use disorder matter in patients with bipolar disorder? J Clin Psychiatry 2005; 66:730-735; quiz 808-809.

40. Keck PE Jr, McElroy SL, Strakowski SM, Stanton SP, Kizer DL, Balistreri TM, et al. Factors associated with pharmacologic noncompliance in patients with mania. J Clin Psychiatry 1996;57:292-297.

41. Weiss RD, Greenfield SF, Najavits LM, Soto JA, Wyner D,
Tohen M, et al. Medication compliance among patients with bipolar disorder and substance use disorder. J Clin Psychiatry 1998;59:172-174.

42. Chandra IS, Kumar KL, Reddy MP, Reddy CM. Attitudes toward medication and reasons for non-compliance in patients with schizophrenia. Indian J Psychol Med 2014;36:294-298.

43. Hall AK, Cole-Lewis H, Bernhardt JM. Mobile text messaging for health: a systematic review of reviews. Annu Rev Public Health 2015;36:393-415.

44. Ershad Sarabi R, Sadoughi F, Jamshidi Orak R, Bahaadinbeigy $\mathrm{K}$. The effectiveness of mobile phone text messaging in improving medication adherence for patients with chronic diseases: a systematic review. Iran Red Crescent Med J 2016;18: e25183.

45. El-Mallakh P, Findlay J. Strategies to improve medication adherence in patients with schizophrenia: the role of support services. Neuropsychiatr Dis Treat 2015;11:1077-1090. 